\title{
Engineering better sleep
}

\author{
Ronald D. Chervin · Joseph W. Burns
}

Received: 2 February 2011/Accepted: 1 April 2011/Published online: 13 April 2011

(C) International Federation for Medical and Biological Engineering 2011

\begin{abstract}
Sleep medicine is a growing field with multidisciplinary origins in physiological monitoring techniques, on which it still largely depends. Collaborations between engineers and sleep specialists offer substantial opportunities to improve on current approaches to diagnosis and assessment of patients with sleep problems. Such collaborations could also prove key to improved fundamental understanding of the pathophysiology that underlies sleep disorders and their adverse impact on the brain, cardiovascular system, and optimal health.
\end{abstract}

Keywords Sleep · Polysomnography - Sleep disorders · Sleep apnea, obstructive $\cdot$ Sleep-disordered breathing . Diagnosis · Assessment $\cdot$ Monitoring $\cdot$ Signal analysis

Obstructive sleep apnea affects at least $2 \%$ of women and $4 \%$ of men, and by some estimates rivals diabetes in prevalence. Prolonged periods of insomnia probably affect $10 \%$ of the population at any given time, and $30 \%$ over the lifetime. Restless legs syndrome also affects up to $10 \%$ of the population. The high prevalence and impact of these and many other sleep disorders have given rapid rise to a new medical field, sleep medicine. This area has drawn clinicians, knowledge, research, and support from several

R. D. Chervin $(\bowtie)$

Department of Neurology and Sleep Disorders Center, University of Michigan, C728 Med Inn Bldg 1500 E. Medical Center Dr, Ann Arbor, MI 48109-5845, USA

e-mail: chervin@umich.edu

J. W. Burns

Michigan Tech Research Institute, Michigan Technological

University, 3600 Green Court, Suite 100,

Ann Arbor, MI 48105, USA more traditional fields, including psychiatry, neurology, pulmonary medicine, pediatrics, endocrinology, and otolaryngology. Important contributions from additional medical areas are recognized at an increasing rate. However, engineering and signal analysis are also central to the new discipline, which in many ways began just over 50 years ago with physiological monitoring that first distinguished rapid eye movement (REM) sleep from nonREM sleep. To this day, sleep medicine still depends heavily on neurophysiological and cardiorespiratory monitoring that have evolved only in limited ways during the past several decades.

Signals monitored during polysomnography in a sleep laboratory typically include the electroencephalogram (EEG), surface electromyogram (EMG), electro-oculogram (EOG), electrocardiogram (ECG), nasal and oral airflow, chest and abdominal excursion, snoring sounds, pulse oximetry, and other variables as needed for particular patients [13]. These signals are recorded by specialized technologists at great time and expense. Once signals are captured, even on modern digital systems, most of the analysis depends on human eyes. Each of about 1,000 thirty-second pages is reviewed several times by technologists who score sleep stages, any pauses in breathing, leg movements, and other features. A physician then reviews the scored recording and completes an interpretation of the study. The entire process can generate charges of a few thousand dollars. Similarly complex daytime nap studies, in the form of the Multiple Sleep Latency Test [16], are sometimes performed after nocturnal polysomnography to provide an objective estimate of daytime sleepiness or to help diagnose narcolepsy.

One of the frustrations with these sleep laboratory assessments, however, is that research repeatedly shows them to be less predictive of key sleep disorders 
outcomes-impact on patients' health-than is usually assumed. For example, the rate of apneas or hypopneas on an overnight polysomnogram, often taken as the most important summary of sleep apnea severity, on average explains only about $11 \%$ of the sleepiness recorded on a Multiple Sleep Latency Test, even though sleepiness is considered one of the most common symptoms of obstructive sleep apnea [3]. Even subjective sleepiness appears to be explained very little, or not at all, by those recorded rates of apneas and hypopneas [4]. Technologistscored and quantified arousals, deep non-REM sleep, REM sleep, stage 1 sleep, overall sleep efficiency, and other polysomnographic measures similarly fail to explain as much of patient comorbidity and treatment outcomes as many physicians and patients would hope [2, 6]. These recurrent observations raise a critical question about the extent to which sleep laboratories record the most salient physiological variables to characterize sleep, or breathing during sleep. The problem is that many years after this issue was already apparent [5], knowledge is still limited when it comes to the physiology responsible for causing sleepiness, cardiovascular consequences, endocrine morbidity, or other outcomes strongly suspected to arise from untreated sleep disorders.

In this setting, and facilitated during the past 15 years by conversion from analog to digital sleep recording systems, efforts to improve the clinical utility of sleep assessments have increasingly involved new collaborations between sleep medicine specialists and engineers. Unfortunately, many medical schools now require no math among prerequisites for admission, and most physicians probably remember little of the mathematics they were once taught. Although progressively larger numbers of engineering undergraduates and masters candidates seem to head toward medical school, few end up applying their skills to clinical problems, at least in sleep medicine.

Potential opportunities for cross-trained individuals, or engineers who collaborate with sleep specialists, appear to be numerous. Laboratory evaluations for sleep problems could be made more accurate, reliable, and predictive to the extent that human assessment could be done by machine in an equally accurate but more repeatable and predictable way. Technological advances may well be able to move evaluation for sleep problems out of an artificial sleep laboratory, and into a home environment, with increased comfort, lower expense, and quicker analysis of results. Many opportunities exist to simplify the plethora of biological signals currently acquired to assess patients' sleep states and sleep disorders. Ultimately, assessment of sleep should be made simple and inexpensive enough to be directly accessible to a lay public now equipped with powerful computers, smart phones, and gadgets of all types [21].
A perfect example of an advance in this direction is provided by the sleepiness index, computed from a bispectrum analysis as described by Swarnkar et al. [23] in this issue of MBEC. Relying on signals from one EEG derivation, instead of the six or more used to stage sleep in full polysomnograms, the authors show that sleep latency can be reasonably approximated from the computed sleepiness index, without use of time-consuming human scoring. The results from a standard Multiple Sleep Latency Test are not yet replicated precisely, and the technique may benefit from additional refinement, testing in larger samples, and trial in different settings. However, the Multiple Sleep Latency Test is also known to be an imperfect gold standard [5]. The best test of a new technology against the Multiple Sleep Latency Test, in assessment of daytime sleepiness, would be side-by-side comparison in prediction of future motor vehicle crashes, or some other real-world outcome of key concern to patients.

Newly engineered signal analysis approaches could not only replace partially subjective assessment techniques, but also enable completely novel types of monitoring that are precluded by reliance on human technologists. Swarnkar et al. note that real-time, automatic computation of the sleepiness index based on a single EEG derivation potentially could be used to monitor for the onset of brief microsleep periods, serving for example to alert a sleepy driver and thereby save lives. Applications could prove even broader: workers might monitor their own cognitive state and alertness, to maximize productivity; or the military might remotely monitor sleepiness among soldiers, judging electronically when rest could be vital.

Perhaps as important in the long-term as more immediate clinical applications, new engineering approaches to assessment of sleep and its disorders could greatly improve insight into the physiological mechanisms that underlie the considerable morbidity that these conditions can cause. A better mathematical understanding of the underlying pathophysiology could generate vital clues on how to prevent consequences of sleep disorders, improve alertness, and thereby maximize cognitive function during wakefulness. For example, computer detection of subtle EEG changes in association with each non-apneic respiratory cycle during sleep [7, 8]—putative inspiratory microarousals-may improve prediction of sleepiness in patients with sleep apnea [9]. At the same time, the important physiological insight is that sleepiness may arise not only from more obvious, 10-second or longer apneas and hypopneas, but also from much more numerous, if subtle effects of labored breathing on the sleeping cerebral cortex [20].

What will be necessary to achieve all this? Already, some engineers have started to tackle many of the above 
problems, challenges, and opportunities in sleep medicine, as evidenced by increasing numbers of reports on these topics in this journal [1, 10-12, 14, 15, 17-19, 22, 24, 25]. However, integration of more advanced math, computer, and physical science training into medical curricula could help advance the cause of sleep medicine in addition to that of many other fields that also depend on physiological time signal analysis. Individuals with undergraduate through graduate-level training in engineering, computing, and biomedical sciences should be encouraged to not only consider medical school, but to create combined careers in academics or industry that would put expensive training to maximal use. Collaborations between engineers and physicians, in sleep medicine in particular, should be encouraged and supported by the NIH and other research funding sources. The final translation of sleep and engineering innovation into products that might improve the lives of so many millions-everyone sleeps suboptimally at least on occasion-could depend on the foresight of the investment community to support early stage, clinically driven ideas that emerge from newly honed research focus, during wakefulness, on the one-third of human life spent asleep.

Acknowledgment This work was support by NIH/NHLBI (HL080941).

\section{References}

1. Abeyratne UR, Karunajeewa AS, Hukins C (2007) Mixed-phase modeling in snore sound analysis. Med Biol Eng Comput 45(8):791-806

2. Bennett LS, Langford BA, Stradling JR, Davies RJO (1998) Sleep fragmentation indices as predictors of daytime sleepiness and nCPAP response in obstructive sleep apnea. Am J Respir Crit Care Med 158:778-786

3. Chervin RD, Aldrich MS (1998) Characteristics of apneas and hypopneas during sleep and relation to excessive daytime sleepiness. Sleep 21:799-806

4. Chervin RD, Aldrich MS (1999) The Epworth sleepiness scale may not reflect objective measures of sleepiness or sleep apnea. Neurology 52:125-131

5. Chervin RD, Guilleminault C (1995) Assessment of sleepiness in clinical practice. Nat Med 1:1252-1253

6. Chervin RD, Kraemer HC, Guilleminault C (1995) Correlates of sleep latency on the multiple sleep latency test in a clinical population. Electroencephalogr Clin Neurophysiol 95:147-153

7. Chervin RD, Burns JW, Subotic NS, Roussi C, Thelen B, Ruzicka DL (2004) Method for detection of respiratory cycle-related EEG changes in sleep-disordered breathing. Sleep 27:110-115

8. Chervin RD, Burns JW, Subotic NS, Roussi C, Thelen B, Ruzicka DL (2004) Correlates of respiratory cycle-related EEG changes in children with sleep-disordered breathing. Sleep 27:116-121
9. Chervin RD, Burns JW, Ruzicka DL (2005) Electroencephalographic changes during respiratory cycles predict sleepiness in sleep apnea. Am J Respir Crit Care Med 171:652-658

10. Choi BH, Seo JW, Choi JM et al (2007) Non-constraining sleep/ wake monitoring system using bed actigraphy. Med Biol Eng Comput 45(1):107-114

11. Cuppens K, Lagae L, Ceulemans B, Van Huffel S, Vanrumste B (2010) Automatic video detection of body movement during sleep based on optical flow in pediatric patients with epilepsy. Med Biol Eng Comput 48(9):923-931

12. Huupponen E, Saunamaki T, Saastamoinen A, Kulkas A, Tenhunen M, Himanen SL (2009) Improved computational frontocentral sleep depth parameters show differences between apnea patients and control subjects. Med Biol Eng Comput 47(1):3-10

13. Iber C, Ancoli-Israel S, Chesson A, Quan SF (2007) For the American Academy of Sleep Medicine. The AASM manual for the scoring of sleep and associated events: rules, terminology and technical specifications. American Academy of Sleep Medicine, Westchester

14. Kulkas A, Rauhala E, Huupponen E et al (2008) Detection of compressed tracheal sound patterns with large amplitude variation during sleep. Med Biol Eng Comput 46(4):315-321

15. Kulkas A, Huupponen E, Virkkala J et al (2009) New tracheal sound feature for apnoea analysis. Med Biol Eng Comput 47(4):405-412

16. Littner MR, Kushida C, Wise M et al (2005) Practice parameters for clinical use of the multiple sleep latency test and the maintenance of wakefulness test-an American Academy of Sleep Medicine report-standards of practice committee of the American Academy of Sleep Medicine. Sleep 28(1):113-121

17. Marcos JV, Hornero R, Alvarez D, del Campo F, Lopez M, Zamarron C (2008) Radial basis function classifiers to help in the diagnosis of the obstructive sleep apnoea syndrome from nocturnal oximetry. Med Biol Eng Comput 46(4):323-332

18. Marcos JV, Hornero R, Alvarez D, Del Campo F, Aboy M (2010) Automated detection of obstructive sleep apnoea syndrome from oxygen saturation recordings using linear discriminant analysis. Med Biol Eng Comput 48(9):895-902

19. Mendez MO, Bianchi AM, Montano N et al (2008) On arousal from sleep: time-frequency analysis. Med Biol Eng Comput 46(4):341-351

20. Paruthi S, Chervin RD (2010) Approaches to the assessment of arousals and sleep disturbance in children. Sleep Med 11(7):622-627

21. Shambroom J, Fabregas SE (2010) Age related changes in objectively measured sleep observed in a large population in the home. Sleep 33:A348

22. Swarnkar V, Abeyratne UR, Hukins C, Duce B (2009) A state transition-based method for quantifying EEG sleep fragmentation. Med Biol Eng Comput 47(10):1053-1061

23. Swarnkar V, Abeyratne U, Hukins C (2010) Objective measure of sleepiness and sleep latency via bispectrum analysis of EEG. Med Biol Eng Comput 48(12):1203-1213

24. Yadollahi A, Giannouli E, Moussavi Z (2010) Sleep apnea monitoring and diagnosis based on pulse oximetry and tracheal sound signals. Med Biol Eng Comput 48(11):1087-1097

25. Yamashiro SM (2007) Non-linear dynamics of human periodic breathing and implications for sleep apnea therapy. Med Biol Eng Comput 45(4):345-356 